\title{
Recent advances in Bayesian optimization with applications to parameter reconstruction in optical nano-metrology
}

\author{
Matthias Plock ${ }^{\mathrm{a}}$, Sven Burger ${ }^{\mathrm{a}, \mathrm{b}}$, and Philipp-Immanuel Schneider ${ }^{\mathrm{a}, \mathrm{b}}$ \\ a Zuse Institute Berlin, Takustraße 7, 14195 Berlin, Germany \\ b JCMwave GmbH, Bolivarallee 22, 14050 Berlin, Germany
}

\begin{abstract}
Parameter reconstruction is a common problem in optical nano metrology. It generally involves a set of measurements, to which one attempts to fit a numerical model of the measurement process. The model evaluation typically involves to solve Maxwell's equations and is thus time consuming. This makes the reconstruction computationally demanding. Several methods exist for fitting the model to the measurements. On the one hand, Bayesian optimization methods for expensive black-box optimization enable an efficient reconstruction by training a machine learning model of the squared sum of deviations $\chi^{2}$. On the other hand, curve fitting algorithms, such as the Levenberg-Marquardt method, take the deviations between all model outputs and corresponding measurement values into account which enables a fast local convergence. In this paper we present a Bayesian Target Vector Optimization scheme which combines these two approaches. We compare the performance of the presented method against a standard Levenberg-Marquardt-like algorithm, a conventional Bayesian optimization scheme, and the L-BFGS-B and Nelder-Mead simplex algorithms. As a stand-in for problems from nano metrology, we employ a non-linear least-square problem from the NIST Standard Reference Database. We find that the presented method generally uses fewer calls of the model function than any of the competing schemes to achieve similar reconstruction performance.
\end{abstract}

Keywords: Bayesian Target Vector Optimization, Bayesian optimization, Nonlinear regression, Gaussian process, Least Squares, Levenberg-Marquardt, L-BFGS-B, Nelder-Mead, inverse problem, parameter reconstruction, optical nano-metrology

This paper will be published in Proc. SPIE Vol. 11783 (2021) 117830J (Modeling Aspects in Optical Metrology VIII, DOI: 10.1117/12.2592266) and is made available as an electronic preprint with permission of SPIE. One print or electronic copy may be made for personal use only. Systematic or multiple reproduction, distribution to multiple locations via electronic or other means, duplication of any material in this paper for a fee or for commercial purposes, or modification of the content of the paper are prohibited.

\section{INTRODUCTION}

A parameter reconstruction in optical nano metrology is usually formulated as an inverse problem. After having recorded experimental measurement data, a parametric numerical model of the measurement process is created. A numerical optimization scheme seeks to minimize the deviation between the experimental and model results by repetitively evaluating the model for different parameter. ${ }^{1-4}$

Depending on the numerical complexity of the model, obtaining the result for any given parameter can take from a few seconds to several hours. This creates the need for efficient and robust reconstruction algorithms that use the available resources most effectively, while still achieving excellent reconstruction results.

Bayesian optimization (BO) methods ${ }^{5,6}$ have gained a reputation for being efficient at optimizing outputs of expensive models. They construct a stochastic surrogate of the model, mostly a Gaussian process (GP), ${ }^{7}$ using the output of all previous model evaluations. At each iteration, the surrogate is used to identify new trial model parameters that maximize for example the expected improvement or the probability of improvement. The approach offers a large degree of flexibility such that many extensions of BO have been proposed to cope, e.g., with a large number of model parameters,${ }^{8,9}$ a large number of observations,${ }^{10}$ or noisy model outputs. ${ }^{11}$

Typically, BO methods are used to optimize scalar functions. In the case of a parameter reconstruction, often the sum of squared deviations between model and measurements $\chi^{2}$ is minimized. Minimizing the scalar $\chi^{2}$ has 
the drawback that the information on the multitude of model outputs is lost. On the other hand, least-square curve fitting methods, such as the Levenberg-Marquardt method, use all value outputs to fit the model to the measurement which allows for a fast local convergence. Recently, Uhrenholt \& Jensen proposed a method to perform such a nonlinear least-square regression using a BO method. ${ }^{12}$

In this paper, we present the application of this Bayesian target vector optimization (BTVO) scheme to a parameter reconstruction problem of the NIST Statistical Reference Database. ${ }^{13}$ This problem poses as a stand-in for actual problems from nano metrology, as it has a comparable number of parameters and degrees of freedom. While Uhrenholt \& Jensen compared their approach to the standard BO method, we extend this analysis in two aspects. Firstly, we consider the case that derivative information of the model is available to improve the efficiency of the parameter reconstruction. Secondly, we compare the performance to other standard algorithms used in metrology such as a Levenberg-Marquardt based least-square minimization, the Nelder-Mead algorithm and the gradient based L-BFGS-B method.

In section 2 we discuss BO methods. We first review a conventional BO scheme and discuss how it can be used to perform a least square regression task. We highlight the issues that can arise in this context. To solve these issues, we introduce the BTVO scheme. In section 3 we discuss the benchmarking procedure to compare the proposed BTVO scheme to other common optimization methods. We introduce the problem for which we reconstruct the model parameters and show the reconstruction results for the different optimization methods. Here, we also show how the availability of derivative information can drastically speed up the parameter reconstruction. Finally, in section 4 , we give a brief overview over possible applications and extensions which are made possible by the new BTVO method.

\section{BAYESIAN OPTIMIZATION METHODS}

Bayesian optimization methods are global optimization methods that work very well for expensive black-box objective functions. In essence they consist of two components: the construction and training of a surrogate model at each step of the optimization, and the maximization of an acquisition function (which in turn uses the surrogate model) that then yields a new parameter $\mathbf{p}_{j+1}$ to sample. The goal of this iterative optimization process is to find a point $\mathbf{p}^{*}$ in the parameter space which maximizes or minimizes the objective function.

\subsection{Conventional Bayesian Optimization}

The conventional BO method is mainly used to optimize scalar functions. At the beginning of the optimization process, a stochastic surrogate model of the objective function $f: \mathcal{X} \subset \mathbb{R}^{M} \rightarrow \mathbb{R}$ is created and then trained with each new observation of $f(\mathbf{p})$. The surrogate model can be typically evaluated much faster than the expensive objective function. A common choice is to use GPs as surrogate models. ${ }^{7}$ A GP is defined by a mean function $m(\mathbf{p})$ and a covariance kernel function $k\left(\mathbf{p}, \mathbf{p}^{\prime}\right)$. Common initial choices, which are also employed in the following, are a constant mean function and a Matérn $5 / 2$ kernel function, ${ }^{14}$

$$
m(\mathbf{p})=m_{0}, \quad \text { and } \quad k\left(\mathbf{p}, \mathbf{p}^{\prime} ; \boldsymbol{\theta}\right)=\sigma_{0}^{2}\left(1+\sqrt{5} r+\frac{5}{3} r^{2}\right) \exp (-\sqrt{5} r) \quad \text { with } \quad r=\left(\sum_{i=1}^{M} \frac{\left(p_{i}-p_{i}^{\prime}\right)^{2}}{l_{i}^{2}}\right)^{1 / 2}
$$

where $m_{0}$ and $\left[\sigma_{0}, l_{1}, \ldots, l_{M}\right]=\boldsymbol{\theta}$ are the hyperparameters of the mean and covariance function, respectively. Given a set of observations $\mathbf{Y}=\left[\mathbf{y}_{1}, \mathbf{y}_{2}, \ldots\right]$ for a set of model parameters $\mathbf{P}=\left[\mathbf{p}_{1}, \mathbf{p}_{2}, \ldots\right]$ and hyperparameters $m_{0}, \boldsymbol{\theta}$, a GP can predict a normal distribution of the function value $\hat{f}(\mathbf{p}) \sim \mathcal{N}\left(\mu(\mathbf{p}), \sigma^{2}(\mathbf{p})\right)$ for each parameter $\mathbf{p} \in \mathcal{X}$ with

$$
\begin{array}{r}
\mu(\mathbf{p})=m_{0}+\mathbf{k}^{T} K^{(-1)}\left(\mathbf{Y}-\mathbf{1} m_{0}\right) \quad \text { and } \quad \sigma^{2}(\mathbf{p})=\sigma_{0}^{2}+\mathbf{k}^{T} K^{(-1)} \mathbf{k}, \\
\text { where } \quad \mathbf{k}=\left[k\left(\mathbf{p}, \mathbf{p}_{1} ; \boldsymbol{\theta}\right), k\left(\mathbf{p}, \mathbf{p}_{2} ; \boldsymbol{\theta}\right), \ldots\right]^{T} \quad \text { and } \quad(K)_{i j}=k\left(\mathbf{p}_{i}, \mathbf{p}_{j} ; \boldsymbol{\theta}\right) .
\end{array}
$$

The choice of the hyperparameter values is essential for obtaining a good prediction. A common approach to optimize their valuesis to maximize the log marginal likelihood,

$$
\log p(\mathbf{Y} \mid \mathbf{P}, \boldsymbol{\theta})=-\frac{1}{2}\left(\mathbf{Y}-\mathbf{1} m_{0}\right)^{T} K^{(-1)}(\mathbf{P} ; \boldsymbol{\theta})\left(\mathbf{Y}-\mathbf{1} m_{0}\right)-\frac{1}{2} \log |K(\mathbf{P} ; \boldsymbol{\theta})|-\frac{n}{2} \log 2 \pi,
$$




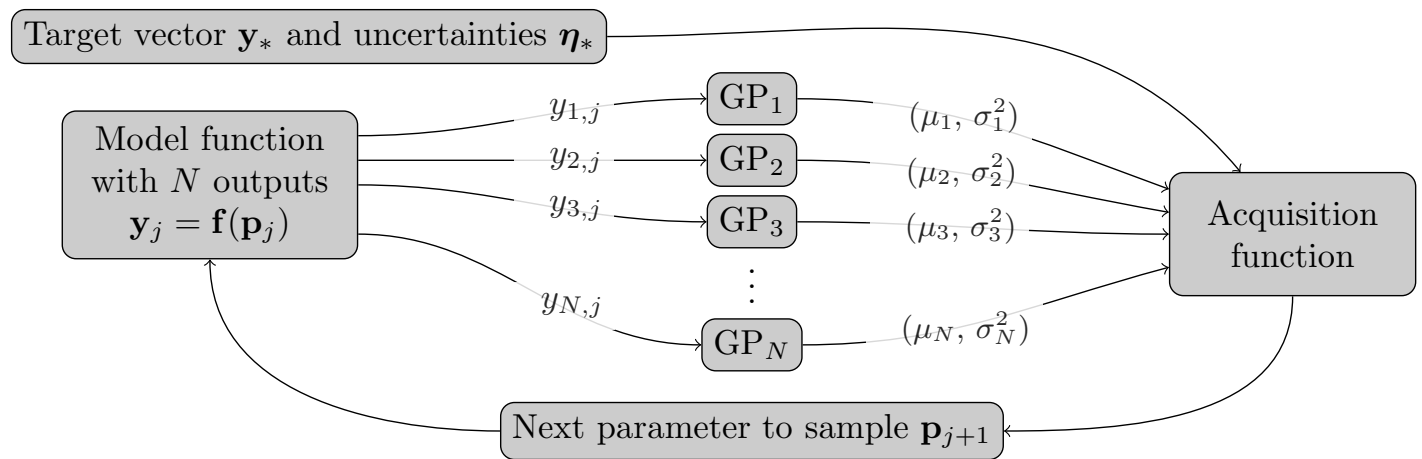

Figure 1: A schematic of the BTVO scheme. Each of the $N$ outputs of the model function is used to construct a surrogate model, in this case Gaussian processes. Each of these $N$ Gaussian processes provides predictions about the mean and variance in the parameter space, which is used by the acquisition function to calculate a new sampling point or evaluation candidate $\mathbf{p}_{j+1}$ for the model function.

of the observed data $\mathbf{Y} .^{7,14}$ The predicted normal distribution is used to identify a new trial parameter set $\mathbf{p}_{j+1}$ for the next iteration of the optimization. To this end, an acquisition function $\alpha(\mathbf{p})$ is maximized, i.e.

$$
\mathbf{p}_{j+1}=\underset{\mathbf{p} \in \mathcal{X}}{\arg \max } \alpha(\mathbf{p})
$$

Common choices of acquisition functions are probability of improvement (PoI), ${ }^{14}$ expected improvement $(\mathrm{EI}),{ }^{5,15}$ and lower- and upper confidence bound (LCB and UCB). ${ }^{16}$

In order to perform a least-square regression with the conventional BO method, we have to scalarize the objective function. This is often done by calculating the reduced $\chi^{2}$ statistic,

$$
\chi_{\mathbf{p}}^{2}=\frac{\left(\mathbf{y}_{*}-\mathbf{f}(\mathbf{p})\right)^{T} H\left(\mathbf{y}_{*}-\mathbf{f}(\mathbf{p})\right)}{N-M} \quad \text { with } \quad H=\operatorname{diag}\left(\boldsymbol{\eta}_{*}^{2}\right)^{-1} .
$$

Here, $\mathbf{y}_{*}$ are the observed data points to which we want to fit the model $\mathbf{f}(\mathbf{p})$, and $\boldsymbol{\eta}_{*}^{2}$ is a vector containing the measurement variance of the observed data points. Typically, the evaluation of the model function $\mathbf{f}(\mathbf{p})$ is computationally expensive, such that it can be beneficial to model $\chi_{\mathbf{p}}^{2}$ by a GP to determine parameter vectors $\mathbf{p}_{j+1}$ that likely lead to a reduction of $\chi_{\mathbf{p}}^{2}$. However this approach has two important disadvantages. First, by modeling $\chi_{\mathbf{p}}^{2}$ with a single GP, most of the information about the $N$ dimensional model output of $\mathbf{f}(\mathbf{p})$ is lost. For example, the reduced $\chi^{2}$ statistic does not change when all differences between observed and modeled data points switch their respective sign. This information is potentially important for the optimization process and the prediction of a next sampling point. And second, GPs are designed to model data that follows a normal distribution. This is not the case for the reduced $\chi^{2}$ statistic, which is always larger or equal to zero. Both of these issues are addressed with the proposed BTVO method.

To mitigate the second problem, one can also consider bijective transformations $\chi_{\mathbf{p}}^{2} \rightarrow g\left(\chi_{\mathbf{p}}^{2}\right)$ that change the distribution of function values. For large $N$, the transformation $g\left(\chi_{\mathbf{p}}^{2}\right)=\left(\chi_{\mathbf{p}}^{2}\right)^{1 / 3}$ leads to approximately normally distributed function values. ${ }^{17}$

\subsection{Bayesian Target Vector Optimization}

To overcome the issues found for a conventional BO approach, Uhrenholt \& Jensen propose to use $N$ individual GPs to model the $N$ outputs of the model function $\mathbf{f}(\mathbf{p})^{12}$ (cf. fig. 1 for a schematic overview), i.e.

$$
\mathbf{y}_{j}=\mathbf{f}\left(\mathbf{p}_{j}\right), \quad \text { and } \quad y_{1, j} \rightarrow \mathrm{GP}_{1}, \quad y_{2, j} \rightarrow \mathrm{GP}_{2}, \quad \ldots, \quad y_{N, j} \rightarrow \mathrm{GP}_{N}
$$

As GPs are a stochastic model of the objective function, each GP defines a normally distributed random variable $\hat{f}_{i}(\mathbf{p}) \sim \mathcal{N}\left(\mu_{i}(\mathbf{p}), \sigma_{i}^{2}(\mathbf{p})\right)$ for each parameter $\mathbf{p}$ in the design space. Using these random variables, one can define 
a random variable

$$
\hat{d}_{i}(\mathbf{p})=\frac{\hat{f}_{i}(\mathbf{p})-y_{*, i}}{\eta_{*, i}}
$$

for each difference from the model prediction to the respective target vector component $y_{*, i}$. Being a linear transformation of the GPs predictions, all $\hat{d}_{i}$ follow a normal distribution, with mean $d_{i}(\mathbf{p})=\left(\mu_{i}(\mathbf{p})-y_{*, i}\right) / \eta_{*, i}$ and variance $\sigma_{i}^{2}(\mathbf{p}) / \eta_{*, i}^{2}$. The predicted residual sum of squares,

$$
\hat{\chi}^{2}(\mathbf{p})=\sum_{i}^{N} \hat{d}_{i}^{2}(\mathbf{p})
$$

then follows the generalized $\chi^{2}$ distribution $G \chi^{2}(\mathbf{w}, \mathbf{N}, \boldsymbol{\lambda})$, with weight vector $\mathbf{w}=\left[\sigma_{1}^{2}(\mathbf{p}) / \eta_{*, 1}^{2}, \ldots, \sigma_{N}^{2}(\mathbf{p}) / \eta_{*, N}^{2}\right]^{T}$, the degree-of-freedom vector $\mathbf{N}=[1, \ldots, 1]^{T}$, and the non-centrality vector $\boldsymbol{\lambda}=\left[\lambda_{1}(\mathbf{p}), \ldots, \lambda_{N}(\mathbf{p})\right]^{T}$, with each vector of length $N$. Here, the non-centrality parameter is defined as $\lambda_{i}(\mathbf{p})=d_{i}^{2}(\mathbf{p})$. Predictions for the residual sum of squares require evaluation of the cumulative distribution function (CDF) of this probability distribution. Unfortunately, no closed form expression exists for the generalized $\chi^{2}$ distribution. Instead Uhrenholt \& Jensen consider the random variable

$$
\tilde{\chi}^{2}(\mathbf{p})=\sum_{i}^{N} \tilde{d}_{i}^{2}(\mathbf{p}) \quad \text { with } \quad \tilde{d}_{i}^{2}(\mathbf{p})=\hat{d}_{i}^{2}(\mathbf{p}) \frac{\eta_{*, i}^{2}}{\sigma_{i}^{2}(\mathbf{p})}
$$

Each rescaled squared distance $\tilde{d}_{i}^{2}(\mathbf{p})$ follows a non-central $\chi^{2}$ distribution $\tilde{d}_{i}^{2}(\mathbf{p}) \sim N C \chi^{2}(1, \lambda(\mathbf{p}))$ with one degree of freedom and non-centrality parameter $\lambda(\mathbf{p})=d_{i}^{2}(\mathbf{p}) \frac{\eta_{*, i}^{2}}{\sigma_{i}^{2}(\mathbf{p})}$. Consequently, $\tilde{\chi}^{2}(\mathbf{p})$ follows a non-central $\chi^{2}$ distribution with $N$ degrees of freedom and non-centrality parameter

$$
\lambda(\mathbf{p})=\gamma^{-2}(\mathbf{p}) \sum_{i}^{N}\left(\frac{\mu_{i}(\mathbf{p})-y_{*, i}}{\eta_{*, i}}\right)^{2} \quad \text { with } \quad \gamma^{2}(\mathbf{p})=\frac{1}{N} \sum_{i}^{N} \frac{\sigma_{i}^{2}(\mathbf{p})}{\eta_{*, i}^{2}} .
$$

The distribution $\gamma^{2}(\mathbf{p}) \tilde{\chi}^{2}(\mathbf{p})$ is then an unbiased approximation of the generalized non-central $\chi^{2}$ distribution of the random variable $\hat{\chi}^{2}(\mathbf{p}) .{ }^{12}$ It has the advantage that its CDF, denoted $F_{N, \lambda}(t)$, is known analytically. In order to compute acquisition functions such as the expected improvement and lower confidence bound efficiently, $F_{N, \lambda}(t)$ is replaced by its very accurate approximation by a Gaussian CDF,

$$
F_{N, \lambda}(t) \approx \Phi\left(\frac{z-\alpha}{\varrho}\right)
$$

where $z=z(t, N, \lambda)$ refers to the random variable of the distribution, $\alpha=\alpha(t, N, \lambda)$ is the mean of the Gaussian $\mathrm{CDF}$ and $\varrho=\varrho(t, N, \lambda)$ is its standard deviation. With these approximations, the evaluations of the acquisition function require a comparable effort as for the conventional BO scheme. However, for large $N$ the computational effort to make predictions from $N$ independent GPs leads to a very large computational effort. To circumvent this issue we propose to use a shared covariance matrix $K$ across all GPs. The details of this approach will be further discussed in a future publication, which is currently in preparation.

\section{BENCHMARK}

In order to show the capabilities of the BTVO, we apply it to the problem of finding the optimal fit parameters in an analytical nonlinear least squares problem. We compare the proposed method against a Levenberg-Marquardt (LM) like scheme ${ }^{18,19}$ (we employ the scipy implementation scipy.optimize.least_squares ${ }^{20}$ ), a conventional Bayesian optimization (BO) method (as described in section 2.1), the L-BFGS-B scheme ${ }^{21}$ and the Nelder-Mead simplex algorithm $(\mathrm{NM}) .^{22}$

The BTVO and LM methods are both capable of natively performing a least squares regression, as they utilize the discrete outputs of the objective function to perform the fit. The BO, L-BFGS-B, and NM methods 


\begin{tabular}{ccc}
\hline Parameter & Certified value & Parameter range \\
\hline$\beta_{1}$ & $699.64 \pm 16.30$ & {$[100,1000]$} \\
$\beta_{2}$ & $5.28 \pm 2.08$ & {$[1,10]$} \\
$\beta_{3}$ & $0.76 \pm 0.20$ & {$[0.1,1]$} \\
$\beta_{4}$ & $1.28 \pm 0.69$ & {$[1,10]$} \\
\hline
\end{tabular}

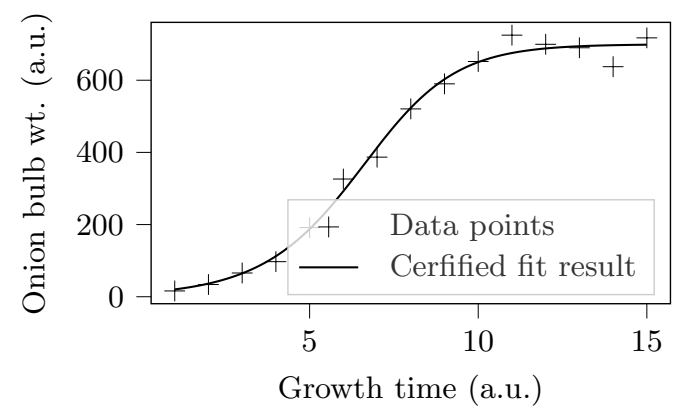

Figure 2: Left: certified values for the Rat43 dataset provided by the NIST Statistical Reference Database, ${ }^{26}$ as well as parameter ranges chosen in the fitting process. Right: data points for the dataset and a certified reconstruction result, with a residual sum of squares $\chi_{\min }^{2}=8786.40 \pm 28.26$. The parameters employed to create the certified fit result are found in the table on the left as certified values.

are usually employed to perform standard optimization tasks, where the minimum or maximum of a function is sought. In order to perform a least squares regression with these schemes, the objective function has to be scalarized first, as is described in section 2.1.

Different optimization methods profit from different scalarization strategies. The L-BFGS-B scheme works very well on quadratic functions, ${ }^{23}$ while the BO implicitly assumes that the objective function is a random number that follows a normal distribution. By taking the third root of the $\chi^{2}$ distributed value eq. (2), we can achieve an approximately normally distributed variable. ${ }^{17}$ Therefore when using the BO method we instead optimize $\left(\chi_{\mathbf{p}}^{2}\right)^{1 / 3}$.

A benchmark consisting of six repeated runs was performed for each optimization method. From these results a mean and standard deviation is calculated, which is shown in the figures as a solid line and a shaded surrounding band of identical color, respectively. The reconstruction algorithms, which are implemented in the analysis and optimization toolbox of the finite element sofware JCMsuite, ${ }^{24}$ were run on 8 cores of a Intel Xeon Gold 6246 CPU cores and $8 \mathrm{~GB}$ of RAM per core.

Bayesian optimization methods can have a substantial computational overhead over other optimization methods. This is because to predict a new sample candidate, several smaller optimization problems have to be solved. This computational overhead may result in BO or BTVO requiring fewer iterations to reconstruct the optimal parameter, but still requiring a longer total runtime. Therefore both, a comparison of the number of iterations (where each iteration is one call to the objective function) and the total computation time are shown.

The metric we choose to measure the performance of the various optimization schemes is the distance $d$ of the reconstruction parameter $\mathbf{p}$ to the optimal reconstruction parameter $\mathbf{p}^{*}$. The distance in each parameter direction is normalized by the standard deviation $\varepsilon_{p_{i}}$ of the reconstruction of each parameter $p_{i}$

$$
d=\sqrt{\sum_{i=1}^{M}\left(\frac{p_{i}-p_{i}^{*}}{\varepsilon_{p_{i}^{*}}}\right)^{2}}
$$

The standard deviations $\varepsilon_{p_{i}^{*}}$ are determined from the Jacobian matrix of $\mathbf{f}(\mathbf{p})$ for $\mathbf{p}=\mathbf{p}^{*} .^{25}$ As a convergence criterion, we consider the case that a distance $d \leq 10^{-1}$ has been reached, i.e. that $\mathbf{p}$ has converged to within $10 \%$ of the reconstruction uncertainty.

\subsection{Problem}

As benchmark problem we choose the Rat43 dataset, which is available in the NIST Standard Reference Database. $^{26,27}$ The dataset contains 15 discrete datapoints which describe the dry weight of onion bulbs and tops as a function of the growing time, as can be seen in fig. 2. The fit model is sigmoidal in nature and is 

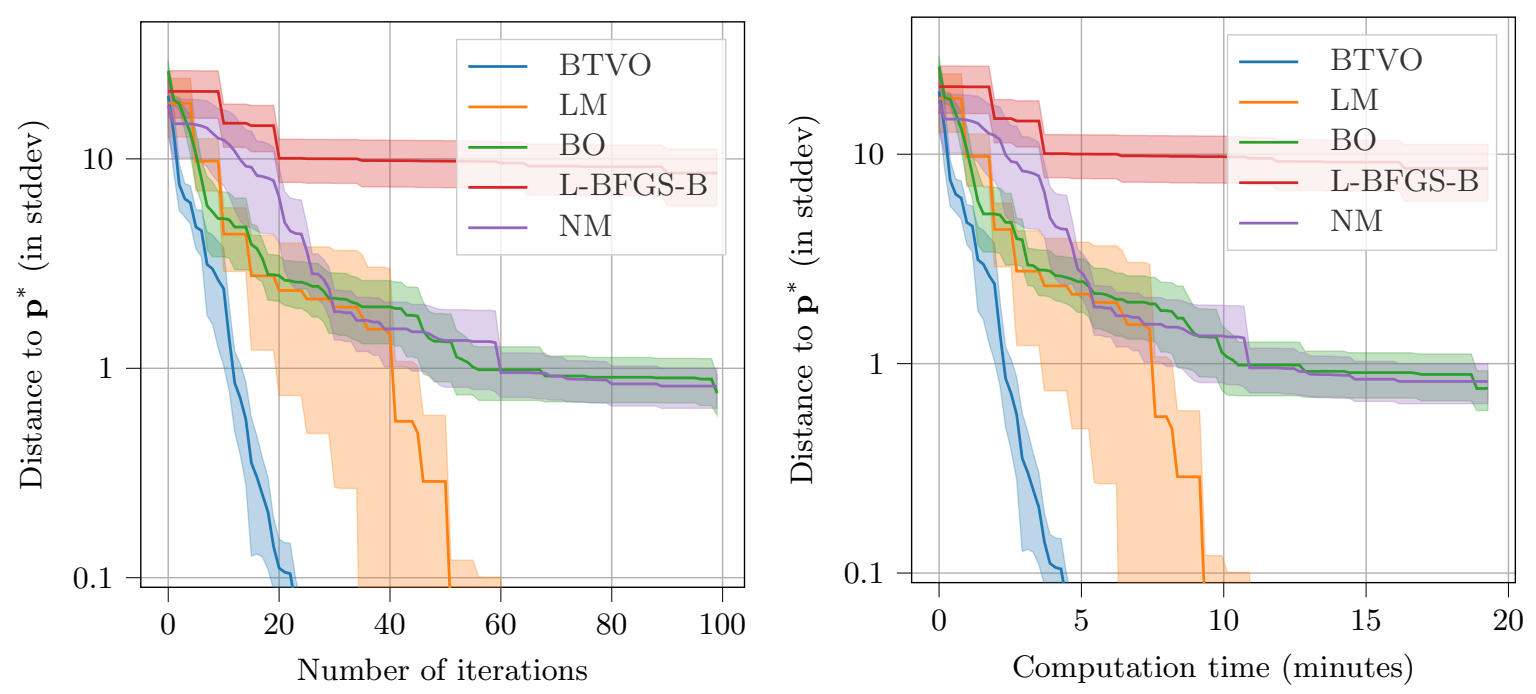

Figure 3: Shown are the results of the different optimization benchmarks: the average reconstruction result as a solid line and its standard deviation as shaded are around the average. The optimization metric is the distance of the reconstructed parameter $\mathbf{p}$ from the optimal parameter $\mathbf{p}^{*}$ in units of the standard deviations of the optimally reconstructed parameter for the BTVO with derivatives, as defined in eq. (3). The proposed BTVO method outperforms every other optimization scheme, despite the computational overhead.

controlled by four parameters which are gathered in the parameter vector $\boldsymbol{\beta}$,

$$
y=f(x, \boldsymbol{\beta})=\frac{\beta_{1}}{1+\exp \left(\beta_{2}-\beta_{3} x\right)^{1 / \beta_{4}}} .
$$

The certified values given in the NIST repository are found in fig. 2. For the optimization a uniform prior with $\beta_{1} \in[100,1000], \beta_{2} \in[1,10], \beta_{3} \in[0.1,1]$ and $\beta_{4} \in[1,10]$ was chosen. The number of datapoints and free parameters is such that this problem can pose as a stand-in for e.g. a small wavelength or angular scan, where a limited number of geometrical and experimental parameters is varied, such as for example in ellipsometry. ${ }^{28}$ Without loss of generality we assume unit variance for each data channel, that is $\eta_{i}^{2}=1 \forall i$. The regressor $x$ is varied between 1 and 15, yielding a vector $\mathbf{y}$ of length 15 .

Advantages of this analytical benchmark approach are the availability of analytical derivatives, the lack of discretization errors of e.g. finite element methods and a very short and controlled actual calculation time.

Due to their computational overhead, BO methods are best employed when used on expensive objective functions. To emulate an expensive model in the chosen analytical problem, a sleep call of ten seconds is inserted into the function evaluation.

\subsection{Reconstruction results}

The results of the different optimization benchmarks are shown in fig. 3. We can clearly separate the performance of the optimization schemes into those which are capable of native least squares regression, and those which require a scalarization of the objective function. The native least squares methods both manage to achieve reconstruction results of $10 \%$ and less, well within the reserved optimization budget. Neither the L-BFGS-B, the NM, nor the conventional BO, are able to reconstruct the model parameters with the same accuracy in the optimization budget.

L-BFGS-B and LM both create a local approximation of the curvature of the objective function. L-BFGS-B uses an averaged approximation of the Hessian of the scalarized objective function, while LM creates a local approximation of the Jacobian of all of the outputs of the objective function. To achieve this, both methods rely on a finite differences scheme. A telltale sign of this is the steps that can be seen in fig. 3 and fig. 4 for 

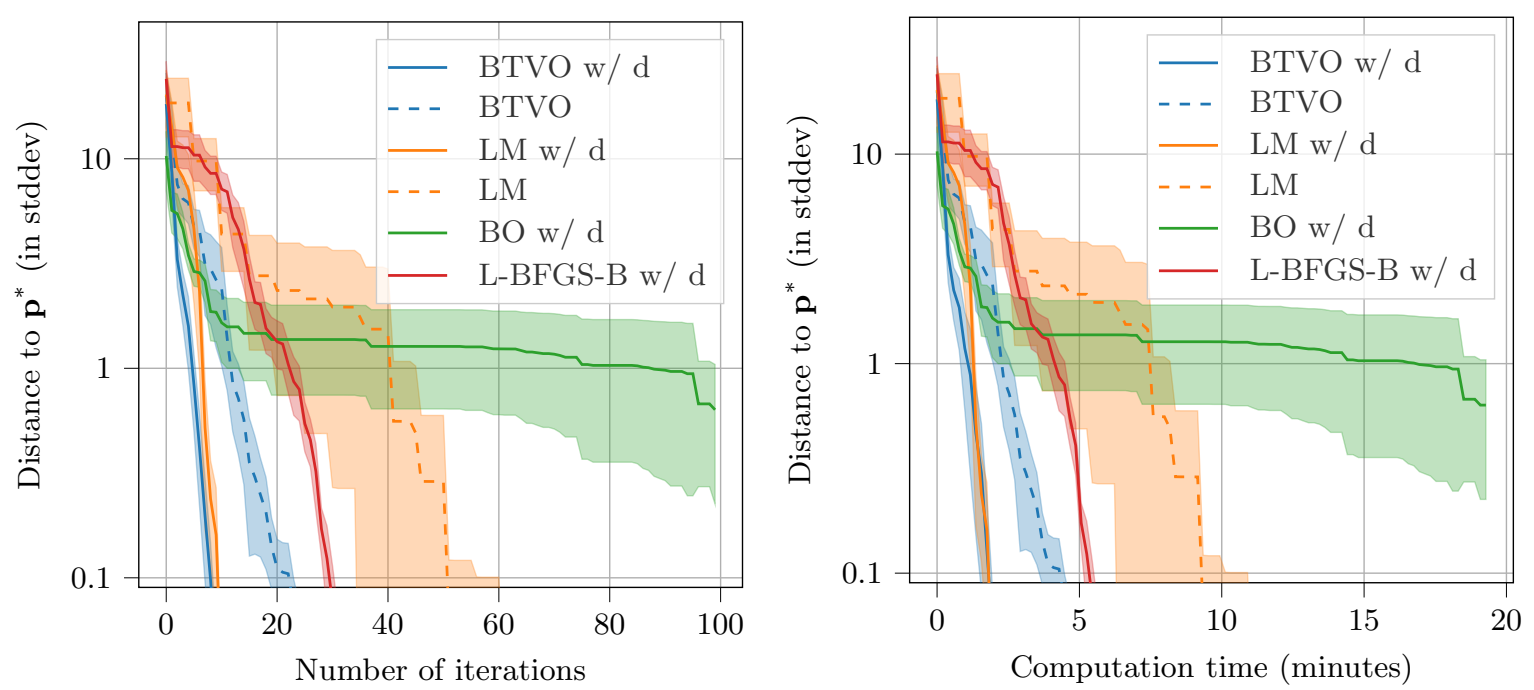

Figure 4: Shown are the results of the different optimization benchmarks or the case that derivative information is available: the average reconstruction result as a solid line and its standard deviation as shaded are around the average. The optimization metric is the distance of the reconstructed parameter $\mathbf{p}$ from the optimal parameter $\mathbf{p}^{*}$ in units of the standard deviations of the optimally reconstructed parameter for the BTVO with derivatives, as defined in eq. (3). If derivatives are available, only the $\mathrm{BO}$ method is not able to reconstruct the model parameters to the desired accuracy.

the two methods. Since L-BFGS-B determines the gradient of the objective function with a finite difference approximation and builds up an aproxiamtion of the Hessian over several iterations, it relies on more inaccurate approximations of the curvature in comparison to the LM approach. This explains the poorer performance of L-BFGS-B for performing a least square regression.

The NM method does not attempt to create an approximation of the local curvature of the objective function. Instead, it places a $M+1$ dimensional simplex in the parameter space, where $M$ is dimensionality of $\mathbf{p}$. The vertices of this simplex are used to create a model of the objective function. At each iteration of the scheme one vertex positions is updated with the goal of converging into a (local) minimizer of the objective. As no iterations are spent for determining finite differences, NM performs better than L-BFGS-B.

The BO method constructs a surrogate model of the scalarized objective function as detailed in section 2.1. At the beginning of the optimization it outperforms NM. After about 30 iterations however, this advantage is lost, and BO and NM perform equally well. Of these three methods, only BO and NM achieve a reconstruction result within one standard deviation, while L-BFGS-B barely manages to reconstruct the parameters within 10 standard deviations. However, as they operate on the scalarized version of the objective function, neither L-BFGS-B, NM, nor BO, are apt methods to solve a least square regression problem.

The two native least squares methods, BTVO and LM, perform much better than the other reconstruction algorithms. They are capable of reconstructing the fit parameters to less than $10 \%$ of the chosen metric. Because LM has to first construct a Jacobian by means of a finite difference scheme, it is at a clear disadvantage over the BTVO. The surrogate model that is constructed during the optimization process with the BTVO containts these higher order terms implicitly. This speeds up the optimization considerably. As such, the BTVO reaches the desired reconstruction result within 24 iterations (corresponding to approximately $4.5 \mathrm{~min}$ ), while the LM algorithm requires 52 iterations $(\approx 9.3 \mathrm{~min})$.

A list of reconstructed parameters is given in table 1. With exception of L-BFGS-B, all reconstructed parameters are well within the given standard deviation given as certified results by the NIST. ${ }^{26}$ 


\begin{tabular}{rrrccccc}
\hline \multicolumn{2}{r}{ Optimization method } & $\beta_{1}$ & $\beta_{2}$ & $\beta_{3}$ & $\beta_{4}$ & \multicolumn{2}{c}{ Mean Its./Time } \\
\hline & BTVO & 699.6 & 5.3 & 0.8 & 1.3 & 24 & $4.5 \mathrm{~min}$ \\
(Without derivatives) & LM & 699.6 & 5.3 & 0.8 & 1.3 & 52 & $9.3 \mathrm{~min}$ \\
& $\mathrm{BO}$ & 702.7 & 4.9 & 0.7 & 1.1 & $\varnothing$ \\
& L-BFGS-B & 668.9 & 7.9 & 1.0 & 2.3 & $\varnothing$ \\
(With derivatives) & NM & 697.5 & 5.8 & 0.8 & 1.4 & $\varnothing$ \\
\hline & BTVO & 699.6 & 5.3 & 0.8 & 1.3 & 9 & $1.9 \mathrm{~min}$ \\
& LM & 699.6 & 5.3 & 0.8 & 1.3 & 11 & $1.9 \mathrm{~min}$ \\
& BO & 699.4 & 5.3 & 0.8 & 1.3 & & $\varnothing$ \\
& L-BFGS-B & 699.6 & 5.3 & 0.8 & 1.3 & 31 & $5.5 \mathrm{~min}$ \\
\hline
\end{tabular}

$\begin{array}{lllll}\text { Certified results } & 699.6 \pm 16.3 & 5.3 \pm 2.1 & 0.8 \pm 0.2 & 1.3 \pm 0.7\end{array}$

Table 1: The reconstructed parameters sorted by reconstruction scheme used. Also shown is the reconstruction performance in terms of the mean number of calls to the objective function (Its.) and mean time that was required (Time) for the reconstruction to achieve the desired accuracy. A discussion and explanations for deviations from the certified values can be found in section 3.2 and section 3.2.1.

\subsubsection{Including derivative information}

Since we are benchmarking an analytical objective function, derivatives with respect the all parameters are available. This can be used to speed up the parameter reconstruction considerably. Note that in some instances, this information may not be accessible, or only for a limited number of model parameters. Therefore the following results represent an idealized situation that is not necessarily found when trying to perform a least squares fit.

In fig. 4 we show the results of the optimization benchmarks for the methods that can directly utilize derivative information. For easy comparison to the derivative-free methods, BTVO and LM without derivatives are listed as well.

Since we provide the derivatives with respect to all parameters directly, LM and L-BFGS-B do not need to calculate a local approximation to the curvature by means of finite differences. Both schemes greatly benefit from the improved accuracy, but especially L-BFGS-B now also manages to reconstruct the model parameters to the desired accuracy, and well within the optimization budget. Also the convergence of BO improves with the use of derivatives. However, the convergence rate degrades after parameters within one standard deviation to the minimum $(d=1)$ have been found. We attribute this behaviour to the fact that the expected improvement becomes very small for $d \lesssim 1$ and thus other parts of the parameter space are probed within the BO framework.

The substantial difference between the reconstruction performance of BTVO and LM has vanished with the availability of direct derivative information. Looking at the iterations that were required, BTVO took 9 and LM 11 calls of the objective function to achieve the desired accuracy. This slight headstart for the BTVO disappears when we consider the total computation time. Here, both methods achieved the desired accuracy in just under 2 min. This is of course due to the computational overhead of the BTVO method.

Comparing these methods to their derivative-free counterparts, the BTVO with derivatives has achieved the desired accuracy in approximately a third of the iterations that it took without derivatives, and LM has managed the same thing in about a fifth of the iterations.

A list of reconstructed parameters is presented in table 1. All reconstructed parameters are well within the given standard deviation given as certified results by the NIST. ${ }^{26}$

\section{SUMMARY AND OUTLOOK}

We have shown that the proposed extension to BO can be successfully employed in the reconstruction of model parameters of an analytical non-linear least-square problem. For a specific test problem, it performs almost twice as good as an established least-square algorithm when no derivatives are available, and at least similarly well if derivatives are at hand. We have also shown that it outperforms the conventional BO, which has been 
successfully employed in the reconstruction of parameters in optical metrology. ${ }^{29,30}$ We are therefore preparing a publication in which we are investigating the applicability of the BTVO to a comparable problem from optical metrology.

Furthermore, we expect that the BTVO method has important advantages for the error estimation of the reconstructed parameters. BTVO is based on a non-linear surrogate model for each of the outputs of the objective function. This surrogate model can be evaluated quickly to predict the fitting error $\chi^{2}(\mathbf{p})$ close to best parameter set $\mathbf{p}^{*}$ with good accuracy. The surrogate can thus be exploited to perform a Markow Chain Monte Carlo (MCMC) sampling of the posterior density. ${ }^{31,32}$ Performing such a sampling of the actual objective function is computationally very expensive and can take many days. ${ }^{33}$ The use of an accurate surrogate model has thus the potential to accelerate MCMC analyses considerably. We plan to extend our current work in this direction.

Further, the proposed BTVO method does not take covariances between the different data channels into account. This could potentially further enhance the reconstruction ability. A possible approach, introduced by Matsui et al., includes the covariance structure into the GPs. ${ }^{34}$ We assume that a corresponding approach could be applied to the proposed BTVO method.

\section{ACKNOWLEDGMENTS}

This project is funded by the German Federal Ministry of Education and Research (BMBF, project number 05M20ZAA, siMLopt, and project number 01IS20080A, SiM4diM). We further acknowledge funding from the German Federal Ministry of Education and Research (BMBF Forschungscampus MODAL, project number 05M20ZBM) and from the German Federal Ministry for Economic Affairs and Energy (BMWi, project number 50WM2067, Optimal-QT).

\section{REFERENCES}

[1] Gross, H., Model, R., Bär, M., Wurm, M., Bodermann, B., and Rathsfeld, A., "Mathematical modelling of indirect measurements in scatterometry," Measurement 39(9), 782-794 (2006).

[2] Silver, R. M., Barnes, B. M., Heckert, A., Attota, R., Dixson, R., and Jun, J., "Angle resolved optical metrology," Proc. SPIE 6922, 590 - 601 (2008).

[3] Pang, L., Peng, D., Hu, P., Chen, D., He, L., Li, Y., Satake, M., and Tolani, V., "Computational metrology and inspection (CMI) in mask inspection, metrology, review, and repair," Adv. Opt. Techn. 1, 299 (2012).

[4] Kumar, N., Petrik, P., Ramanandan, G. K. P., Gawhary, O. E., Roy, S., Pereira, S. F., Coene, W. M. J., and Urbach, H. P., "Reconstruction of sub-wavelength features and nano-positioning of gratings using coherent fourier scatterometry," Opt. Express 22(20), 24678-24688 (2014).

[5] Močkus, J., "On Bayesian methods for seeking the extremum," in [Optimization techniques IFIP technical conference], 400-404, Springer (1975).

[6] Močkus, J., [Bayesian approach to global optimization: theory and applications], vol. 37, Springer Science \& Business Media (2012).

[7] KI Williams, C., [Gaussian processes for machine learning], Taylor \& Francis Group (2006).

[8] Wang, Z., Zoghi, M., Hutter, F., Matheson, D., De Freitas, N., et al., "Bayesian Optimization in High Dimensions via Random Embeddings," in [IJCAI], 1778-1784 (2013).

[9] Titsias, M. and Lawrence, N. D., "Bayesian Gaussian process latent variable model," in [Proceedings of the Thirteenth International Conference on Artificial Intelligence and Statistics], 844-851, JMLR Workshop and Conference Proceedings (2010).

[10] Garcia-Santiago, X., Burger, S., Rockstuhl, C., and Schneider, P. I., "Bayesian Optimization With Improved Scalability and Derivative Information for Efficient Design of Nanophotonic Structures," Journal of Lightwave Technology 39(1), 167-177 (2021).

[11] Letham, B., Karrer, B., Ottoni, G., Bakshy, E., et al., "Constrained Bayesian optimization with noisy experiments," Bayesian Analysis 14(2), 495-519 (2019).

[12] Uhrenholt, A. K. and Jensen, B. S., "Efficient Bayesian optimization for target vector estimation," in [The 22nd International Conference on Artificial Intelligence and Statistics], 2661-2670, PMLR (2019). 
[13] NIST, "Standard Reference Database," (2003). URL: https://www.itl.nist.gov/div898/strd/index.html (version: 2021-04-20).

[14] Brochu, E., Cora, V. M., and De Freitas, N., "A tutorial on Bayesian optimization of expensive cost functions, with application to active user modeling and hierarchical reinforcement learning," arXiv preprint arXiv:1012.2599 (2010).

[15] Jones, D. R., Schonlau, M., and Welch, W. J., "Efficient global optimization of expensive black-box functions," Journal of Global optimization 13(4), 455-492 (1998).

[16] Auer, P., "Using confidence bounds for exploitation-exploration trade-offs," Journal of Machine Learning Research 3(Nov), 397-422 (2002).

[17] Hawkins, D. M. and Wixley, R., "A note on the transformation of chi-squared variables to normality," The American Statistician 40(4), 296-298 (1986).

[18] Levenberg, K., "A method for the solution of certain non-linear problems in least squares," Quarterly of applied mathematics 2(2), 164-168 (1944).

[19] Marquardt, D. W., "An algorithm for least-squares estimation of nonlinear parameters," Journal of the society for Industrial and Applied Mathematics 11(2), 431-441 (1963).

[20] Virtanen, P., Gommers, R., Oliphant, T. E., Haberland, M., Reddy, T., Cournapeau, D., Burovski, E., Peterson, P., Weckesser, W., Bright, J., van der Walt, S. J., Brett, M., Wilson, J., Millman, K. J., Mayorov, N., Nelson, A. R. J., Jones, E., Kern, R., Larson, E., Carey, C. J., Polat, İ., Feng, Y., Moore, E. W., VanderPlas, J., Laxalde, D., Perktold, J., Cimrman, R., Henriksen, I., Quintero, E. A., Harris, C. R., Archibald, A. M., Ribeiro, A. H., Pedregosa, F., van Mulbregt, P., and SciPy 1.0 Contributors, "SciPy 1.0: Fundamental Algorithms for Scientific Computing in Python," Nature Methods 17, 261-272 (2020).

[21] Byrd, R. H., Lu, P., Nocedal, J., and Zhu, C., "A limited memory algorithm for bound constrained optimization," SIAM Journal on scientific computing 16(5), 1190-1208 (1995).

[22] Nelder, J. A. and Mead, R., "A simplex method for function minimization," The computer journal 7(4), 308-313 (1965).

[23] Liu, D. C. and Nocedal, J., "On the limited memory BFGS method for large scale optimization," Mathematical programming 45(1), 503-528 (1989).

[24] JCMwave, "JCMsuite." URL: https://jcmwave.com/jcmsuite (version 4.4.4, May 10., 2021).

[25] Henn, M.-A., Gross, H., Scholze, F., Wurm, M., Elster, C., and Bär, M., "A maximum likelihood approach to the inverse problem of scatterometry," Opt. Express 20, 12771-12786 (Jun 2012).

[26] NIST Standard Reference Database, "Rat43 dataset," (1983). https://www.itl.nist.gov/div898/strd/nls/data/ratkowsky3.shtml (version: 2021-04-13).

[27] Ratkowsky, D., "Nonlinear regression modelling," (1983).

[28] Afraites, L., Hazart, J., and Schiavone, P., "Application of the Kriging method to the reconstruction of ellipsometric signature," Microelectronic Engineering 86(4-6), 1033-1035 (2009).

[29] Andrle, A., Hönicke, P., Schneider, P., Kayser, Y., Hammerschmidt, M., Burger, S., Scholze, F., Beckhoff, B., and Soltwisch, V., "Grazing incidence x-ray fluorescence based characterization of nanostructures for element sensitive profile reconstruction," in [Modeling Aspects in Optical Metrology VII], 11057, 110570M, International Society for Optics and Photonics (2019).

[30] Schneider, P.-I., Garcia Santiago, X., Soltwisch, V., Hammerschmidt, M., Burger, S., and Rockstuhl, C., "Benchmarking five global optimization approaches for nano-optical shape optimization and parameter reconstruction," ACS Photonics 6(11), 2726-2733 (2019).

[31] Klauenberg, K. and Elster, C., "Markov chain Monte Carlo methods: an introductory example," Metrologia 53(1), S32 (2016).

[32] Farchmin, N., Hammerschmidt, M., Schneider, P.-I., Wurm, M., Bodermann, B., Bär, M., and Heidenreich, S., "Efficient Bayesian inversion for shape reconstruction of lithography masks," Journal of Micro/Nanolithography, MEMS, and MOEMS 19(2), 024001 (2020).

[33] Rasmussen, K., Kondrup, J. B., Allard, A., Demeyer, S., Fischer, N., Barton, E., Partridge, D., Wright, L., Bär, M., Fiebach, H., et al., "Novel mathematical and statistical approaches to uncertainty evaluation: best practice guide to uncertainty evaluation for computationally expensive models," tech. rep., Tech. rep.(Euramet, 2015) (2015). 
[34] Matsui, K., Kusakawa, S., Ando, K., Kutsukake, K., Ujihara, T., and Takeuchi, I., "Bayesian Active Learning for Structured Output Design," arXiv preprint arXiv:1911.03671 (2019). 